\title{
Polyfolio : un outil numérique pour le suivi, l'évaluation et la présentation des compétences de l'ingénieur en formation à Polytechnique Montréal
}

\section{Polyfolio: Online tool for tracking, assessing, and presenting engineering students skills' at Polytechnique Montreal}

\section{Polyfolio: una herramienta digital para el seguimiento, la evaluación y la presentación de las competencias del ingeniero en formación en Polytechnique Montréal}

https://doi.org/10.52358/mm.vi9.250

Anastassis Kozanitis, professeur

Université du Québec à Montréal, Canada

kozanitis.anastassis@uqam.ca

Patrice Farand, maitre d'enseignement

Polytechnique Montréal, Canada

p.farand@polymtl.ca

Yves Boudreault, professeur

Polytechnique Montréal, Canada

yves.boudreault@polymtl.ca 
RÉSUMÉ

Polytechnique Montréal s'est doté d'un portfolio numérique pour faire le suivi et l'évaluation des 12 compétences requises par le Bureau canadien d'agrément des programmes de génie. Cet article présente le contexte ayant mené à la mise en place de l'outil, nommé Polyfolio, ses principales fonctionnalités, ainsi que son processus de conception.

Mots-clés : portfolio, évaluation, compétences, génie

\section{ABSTRACT}

Polytechnique Montréal has developed a digital portfolio to monitor and evaluate the twelve skills the Canadian Board of Accreditation for engineering programs requires. This article presents the context leading to the implementation of the tool, named Polyfolio, its main features, and the design process.

Keywords: portfolio, assessment, skills, engineering

RESUMEN

Polytechnique Montréal ha desarrollado un portafolio digital para monitorear y evaluar las doce competencias requeridas por la Junta Canadiense de Acreditación de Programas. Este artículo presenta el contexto de creación de la herramienta, denominada Polyfolio, sus principales características, así como su proceso de diseño.

Palabras clave: porfolio, evaluación, competencias, ingeniería

\section{Mise en contexte du Polyfolio}

Polytechnique Montréal, université canadienne d'ingénierie, accueille plus de 9000 étudiants aux 3 cycles universitaires, dont plus de 5000 au baccalauréat répartis dans 12 spécialités d'ingénierie. Ces spécialités mènent, au terme de 120 crédits suivis sur 4 ans, à un diplôme d'ingénieur reconnu par les ordres des ingénieurs canadiens, permettant ainsi un accès à la profession. Pour être en mesure d'obtenir cette reconnaissance, chacune des spécialités doit obtenir un agrément du Bureau canadien d'agrément des programmes de génie (BCAPG), bureau qui relève d'Ingénieurs Canada, en faisant la démonstration qu'elle respecte plusieurs normes.

L'une des normes les plus importantes est d'être en mesure de démontrer que les diplômés d'un programme possèdent les compétences requises pour exercer la profession d'ingénieur. À cet effet, le BCAPG a mis en place, à la fin des années 2000, un référentiel de 12 grandes compétences (que le BCAPG nomme "qualités ") à développer dans toutes les facultés de génie au Canada (Ingénieurs Canada, 2018). Le tableau 1 présente la liste de ces 12 compétences. 


\section{Tableau 1}

Compétences à développer chez tous les étudiants en génie au Canada

\begin{tabular}{ll}
\hline 1. Connaissances en génie & 7. Communication \\
2. Analyse de problèmes & 8. Professionnalisme \\
3. Investigation & 9. Impact du génie sur la société et l'environnement \\
4. Conception & 10. Déontologie et équité \\
5. Utilisation d'outils d'ingénierie & 11. Économie et gestion de projets \\
6. Travail individuel et en équipe & 12. Apprentissage continu \\
\hline
\end{tabular}

De plus, chaque programme doit démontrer que les résultats obtenus de l'évaluation de ces compétences permettent de perfectionner la formation offerte. II est donc requis de mettre en place un processus d'amélioration continue. Pour Polytechnique Montréal, ce processus compte cinq grandes étapes (voir figure 1).

\section{Figure 1}

Vision globale du processus d'amélioration continue des 12 compétences de l'ingénieur à Polytechnique Montréal

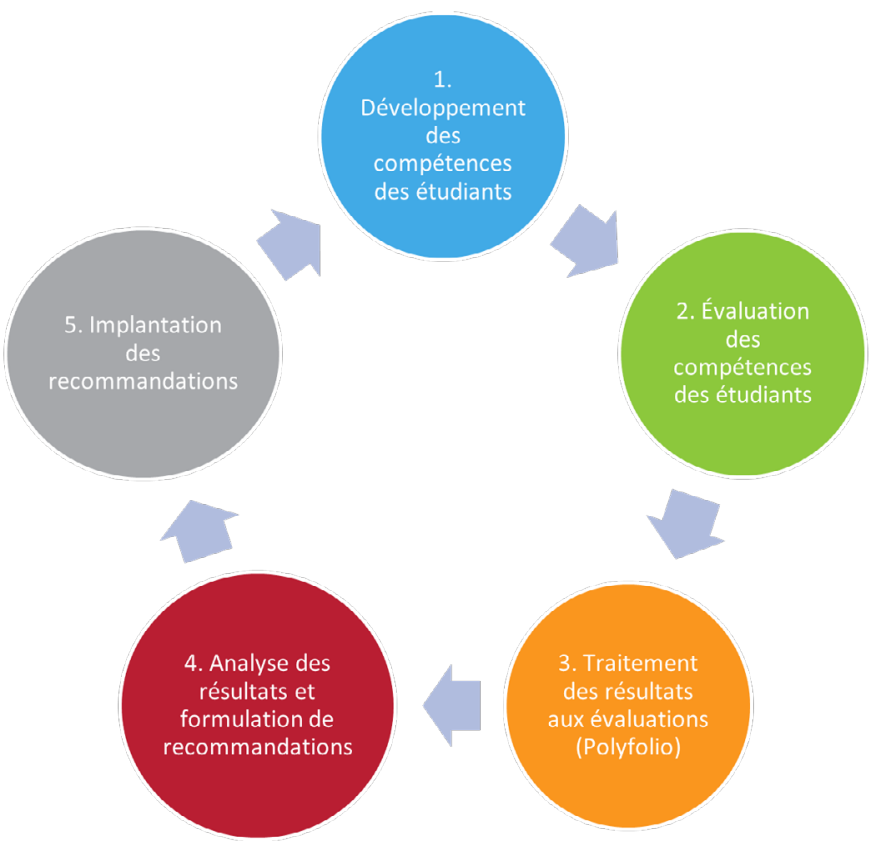


Tout d'abord, le développement des compétences des étudiants (étape 1) s'effectue lors des différentes activités vécues par ces derniers (cours, projets, stages...). Des points de contrôle précis au nombre minimal de trois par compétence ont été identifiés pour évaluer les compétences (étape 2). Pour chacun des points de contrôle, des grilles d'évaluation avec échelles descriptives ont été développées. Les résultats de l'évaluation sont insérés dans un environnement numérique et des graphiques sont générés pour les présenter (étape 3). Ces résultats sont analysés par chacun des programmes, puis des recommandations sont formulées (étape 4). Finalement, les recommandations sont implantées par le programme et les retombées sur le développement des compétences des étudiants sont évaluées (étape 5). Ce cycle se poursuit de façon perpétuelle pour assurer que soit possible l'amélioration continue des programmes.

Pour parvenir à faire le suivi, l'évaluation, la certification et la présentation des compétences des étudiants, il a été nécessaire de développer un portfolio numérique (Bélisle et al., 2017). Cet environnement, nommé Polyfolio, est la pierre angulaire de tout le processus énoncé précédemment et est décrit en détail dans cet article.

\section{Enjeux liés au choix d'un portfolio numérique}

Le recours au portfolio numérique implique un processus de décision qui tient compte des besoins et du contexte de formation et d'évaluation (Shaklee et al., 1997). Pour Polytechnique Montréal, ce processus s'inscrit dans un contexte d'évaluation de compétences et le besoin de documenter le parcours de développement de ces dernières (Tardif, 2006). Ainsi, après avoir considéré des solutions alternatives libres (Mahara et Eduportfolio) ou commerciales (Blackboard Learn, WEAVEOnline et Taskstream), Polytechnique Montréal a fait le choix de développer son propre outil numérique qui sert aujourd'hui de portfolio pour l'ensemble des étudiants inscrits au baccalauréat.

Plusieurs facteurs ont influencé cette décision, notamment des considérations juridiques, économiques et fonctionnelles, ainsi que les injonctions émises par le BCAPG. Sur le plan juridique, les lois canadiennes concernant le droit à la vie privée et la protection des données sensibles et personnelles requièrent que les documents déposés dans le portfolio soient hébergés sur des serveurs situés au Canada. Or, la plupart des solutions commerciales étaient situées aux États-Unis. Par ailleurs, ces dernières avaient des coûts d'acquisition et d'exploitation qui excédaient les limites de l'enveloppe budgétaire allouée par la direction. Toutefois, ce sont les besoins en fonctionnalités ainsi que les prescriptions du BCAPG qui ont le plus influé sur la décision et qui ont conséquemment fait que les solutions alternatives libres, disponibles à l'époque, soient écartées. De surcroît, l'environnement numérique devait être sécurisé pour empêcher que quiconque puisse y déposer un document inapproprié ou encore qu'il puisse modifier les informations consignées de façon frauduleuse. L'enjeu de la validité et de la pérennité des informations contenues ainsi que celui du contrôle des droits d'accès selon le type d'utilisateur se trouvaient au cœur des réflexions préliminaires du processus de conception du portfolio. Ce dernier a nécessité la mise sur pied d'un comité formé d'une gestionnaire de projet, d'un programmeur, du directeur des études de premier cycle, d'un conseiller pédagogique et de trois professeurs. La première tâche du comité a été de rédiger le cahier des charges, un document qui a guidé le travail de conception du programmeur. Le cahier des charges indique les exigences et les spécificités attendues en termes de fonctionnalités désirées, les contraintes évoquées, ainsi que les rôles et les privilèges des usagers. En ce qui a trait aux rôles possibles des usagers, Polyfolio en distingue cinq, soit administrateur, responsable de programme, enseignant, étudiant et représentant BCAPG. 
Pour les étudiants et les enseignants, Polyfolio se veut d'abord un portfolio d'évaluation (Bélair et Van Nieuwenhoven, 2010), c'est-à-dire qu'il permet de faire le suivi et l'évaluation du développement des compétences listées dans le référentiel des programmes de génie. Pour les finissants, il peut également servir de portfolio de présentation auprès d'éventuels employeurs (Bibeau, 2007). Pour les responsables de programme et les représentants du BCAPG, il sert pour l'évaluation et l'amélioration de la qualité des programmes, notamment lors des cycles d'agrément des programmes.

\section{Description de l'environnement numérique Polyfolio}

L'architecture de Polyfolio et son organisation s'articulent autour des 12 compétences. La base de données dynamique qui la sous-tend permet ainsi d'associer les activités liées au développement, au suivi et à l'évaluation des compétences aux différents acteurs impliqués selon leur rôle. Un tableau de bord offre une vue d'ensemble des activités à réaliser durant la formation. Ces activités se réalisent à l'intérieur des cours siglés qui sont obligatoires dans les programmes. Elles consistent pour la plupart en des activités servant à l'évaluation que l'on a nommées « contrôle des acquis ». Les prochains paragraphes présentent des captures d'écran montrant à quoi ressemblent les principales fonctionnalités de Polyfolio. Dans un souci de concision, la description concernera principalement le rôle du responsable de programme, puisqu'il détient les fonctionnalités des autres rôles, sauf pour le rôle de l'administrateur, qui lui assigne les rôles. On présente également la vue du tableau de parcours des étudiants.

\section{Vue responsable de programme}

Le principal rôle du responsable de programme est de créer le plan de son programme pour chaque trimestre. Ce plan contient les points de contrôle des acquis, qui sont habituellement associés à un sigle de cours. Les contrôles des acquis sont listés selon la ou les compétences évaluées et l'année universitaire concernée. Une fois créés, ils sont nommés du nom du sigle du cours concaténé au trimestre concerné, par exemple GCH3515/A2021. II s'agit en fait d'un hyperlien permettant d'y accéder. Le symbole C précise qu'il s'agit d'un contrôle final ou certificatif (voir figure 2). Lorsque le point de contrôle des acquis est créé, les sections (c.-à-d. les groupes) d'un même cours sont créées automatiquement par Polyfolio, puisqu'il est relié au système de gestion académique (GEADE). Les listes d'étudiants inscrits dans les cours sont donc automatiquement créées. 


\section{Figure 2}

Exemple de plan de programme

\begin{tabular}{|c|c|c|c|c|c|c|}
\hline \multicolumn{2}{|c|}{ II Tableau de bord Opérations. } & \multicolumn{4}{|l|}{ Trouver un étudiant } & \& Patrice Farand . \\
\hline \multicolumn{7}{|c|}{ Tableau de bord } \\
\hline Plan & Points de contrôle Zone CÉO & \multicolumn{3}{|c|}{ Trimestre actuel } & & \\
\hline \multicolumn{7}{|c|}{ ๑ Créer un contrôle } \\
\hline \multicolumn{5}{|c|}{ Génie chimique | Automne 2020} & \multicolumn{2}{|c|}{ Trimestre Automne 2020 V } \\
\hline \multicolumn{7}{|c|}{ Plan de programme } \\
\hline \multicolumn{3}{|c|}{ Qualités } & Année 1 & Année 2 & Année 3 & Année 4 \\
\hline & Connaissances en génie & & $\begin{array}{l}\text { GCH1110/A2020 } \\
\text { GCH1511/A2020 }\end{array}$ & & & \\
\hline 02 & Analyse de problèmes & & GCH1110Q2/A2020 & GCH2120/A2020 & GCH3515/A2020 C & \\
\hline 03 & Investigation & & & & & \\
\hline 04 & Conception & & GCH1140Q4/A2020 & & GCH3110/A2020 & GCH8272/A2020 C \\
\hline 05 & Utilisation d'outils d'ingéni & erie & & & & GCH8271Q5/A2020 C \\
\hline & Travail individuel et en équipe & & GCH1140HPR/A2020 & & $\begin{array}{l}\text { GCH3100HPR/A2020 } \mathrm{C} \\
\text { GCH3100CQ6/A2020 }\end{array}$ & \\
\hline 07 & Communication & & $\begin{array}{l}\text { GCH1122Q7/A2020 } \\
\text { GCH3000I/A2020 } \\
\text { GCH3000A/A2020 }\end{array}$ & & $\begin{array}{l}\text { GCH3000CÉO/A2020 C } \\
\text { GCH3000/A2020 }\end{array}$ & \\
\hline 08 & Professionnalisme & & GCH1122Q8/A2020 & & SSH3501/A2020 C & GCH8272Q8/A2020 C \\
\hline 09 & Impact du génie sur la société & et l'environnement & GCH1220/A2020 & & & GCH8272Q9/A2020 C \\
\hline & Déontologie et équité & & & & $\begin{array}{l}\text { SSH3501/A2020 C } \\
\text { GCH3100Q10/A2020 C }\end{array}$ & \\
\hline & Économie et gestion de projet & & $\begin{array}{l}\text { GCH1140E11/A2020 } \\
\text { GCH1140G11/A2020 }\end{array}$ & & GCH3100Q11/A2020 C & $\begin{array}{l}\text { SSH3201-11/A2020 C } \\
\text { SSH3201/A2020 }\end{array}$ \\
\hline & Apprentissage continu & & $\mathrm{GCH} 1122 \mathrm{Q} 12 / \mathrm{A} 2020$ & & $\begin{array}{l}\text { 3100Q12DOC/A2020 C } \\
\text { 3100Q12RéS/A2020 }\end{array}$ & \\
\hline
\end{tabular}

La figure 3 présente la fiche servant à créer un contrôle des acquis et montre les paramètres à préciser. II s'agit du sigle du cours, du trimestre, de la ou des compétences en précisant le niveau de développement lors de cette évaluation et le niveau de développement en fin de parcours, le statut certificatif et le type de grille. Le titre et la description du contrôle sont facultatifs. II est important de mentionner que les tâches utilisées pour évaluer les compétences sont de natures authentiques et proposent des problèmes ou des situations complexes à résoudre. 


\section{Figure 3}

Création d'un contrôle des acquis

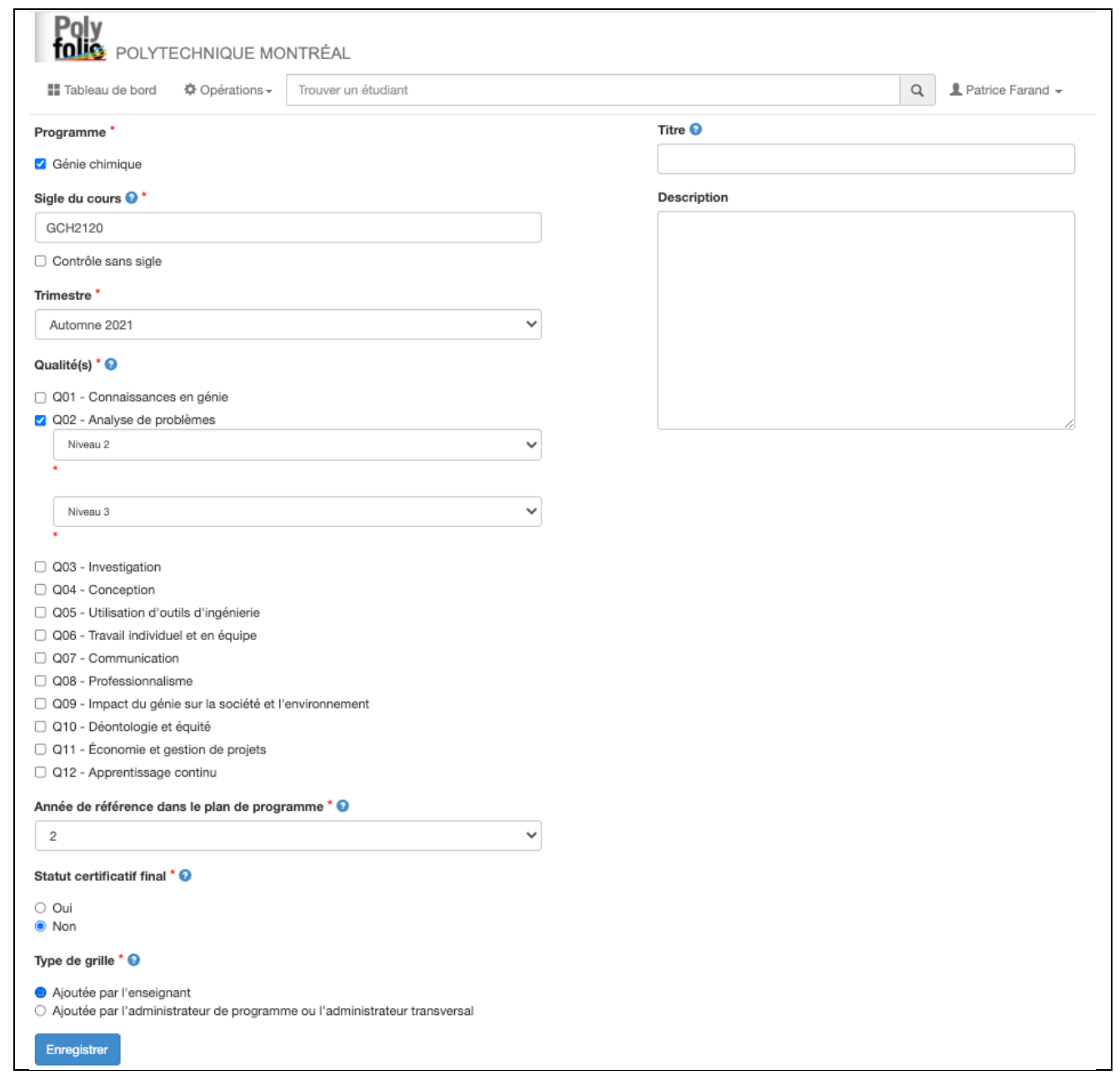

Les niveaux de développement renvoient à une échelle composée de cinq échelons d'autonomie et de complexité croissante en lien avec les situations d'évaluation. Cette échelle a été créée pour servir de référence explicite et commune à l'ensemble des programmes, et permet d'établir une progression graduelle du développement des compétences (voir tableau 2). Ce sont les programmes qui déterminent les échelons intermédiaires et finaux pour chacune des compétences, habituellement choisis entre l'échelon un et quatre. L'échelon cinq ne peut être choisi, car il représente un ingénieur sénior, c'est-à-dire ayant plus de six années d'expérience et le droit de signer des plans et devis. II a été inclus pour marquer la borne supérieure du degré de développement des compétences. Ainsi, les échelons aident les enseignants à déterminer le contexte et les conditions de réalisation des tâches d'apprentissage et d'évaluation. Dans une approche-programme, il est plus aisé de déterminer une progression cohérente des niveaux attendus, puisque les décisions sont prises de manière concertée (Prégent et al., 2009). En effet, selon ces auteurs, l'approche-programme permet des conditions qui facilitent un alignement pédagogique optimal entre les intentions de formation du programme, les activités d'apprentissage et d'évaluation. 


\section{Tableau 2}

Échelle d'autonomie pour l'acquisition et le développement des compétences

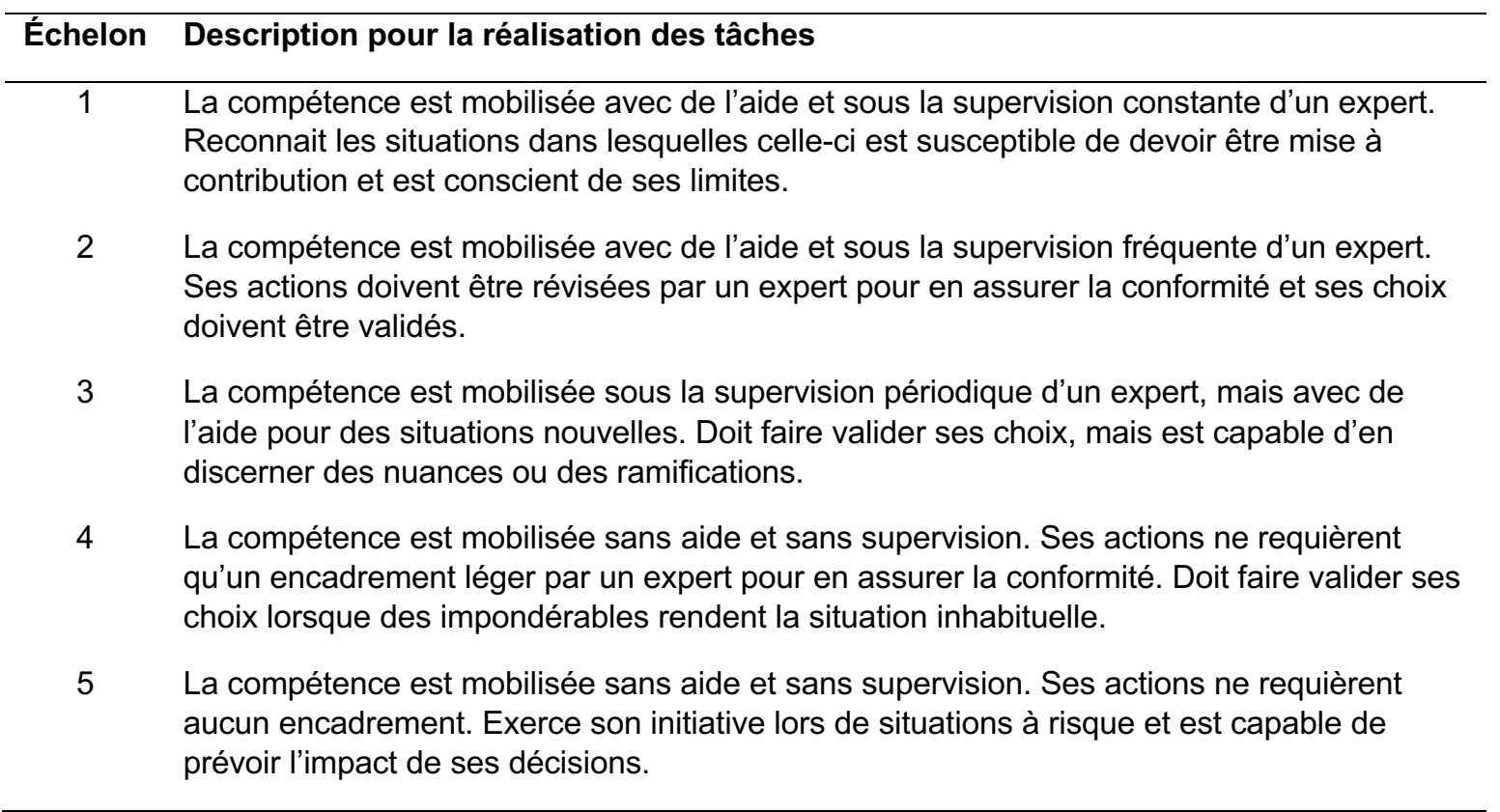

\section{Vue de l'enseignant}

Le principal rôle des enseignants est d'évaluer les compétences à l'intérieur des points de contrôle des acquis, notamment à l'aide de grilles d'évaluation. Ils doivent ainsi créer une grille d'évaluation lorsque le responsable du programme leur en a donné la possibilité. Une grille est créée en précisant les compétences en cohérence avec le contrôle, le mode d'évaluation (individuelle ou équipe), le système de points (10, 20 ou 100) et la diffusion (privée ou publique). La figure 3 montre le résultat une fois l'information saisie. L'enseignant peut également choisir les éléments de compétence qu'il veut évaluer. Ces éléments correspondent aux déclinaisons de la compétence, ils aident à préciser l'essence de la compétence. C'était d'ailleurs une exigence du BCAPG que chaque programme de génie rédige un nombre adéquat d'éléments de compétence, généralement de trois à six, selon la compétence. À ce sujet, Polytechnique a décidé que les éléments de compétence seraient les mêmes pour tous ses programmes. 


\section{Figure 3}

Création d'une grille d'évaluation

\begin{tabular}{|c|c|c|c|}
\hline \multicolumn{4}{|c|}{ Poly } \\
\hline \# Tableau de bord Opérations - & Trouver un étudiant & a & 2 Yves Boudreault - \\
\hline \multicolumn{4}{|c|}{ Tableau de bord / Banque de grilles / Création } \\
\hline \multicolumn{4}{|l|}{ Créer une grille } \\
\hline \multicolumn{4}{|c|}{ Pour débuter, vous devez compléter le formulaire en indiquant quel type de grille que vous désirez créer. } \\
\hline \multicolumn{4}{|c|}{ 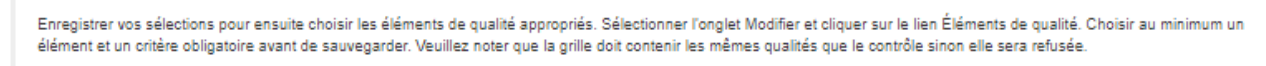 } \\
\hline \multicolumn{4}{|c|}{ 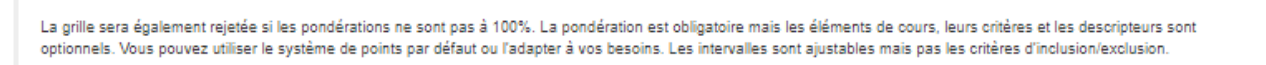 } \\
\hline \multicolumn{4}{|l|}{ Titre de la grille * } \\
\hline \multicolumn{4}{|l|}{ Grille pour le contrôle GCH2525/A2020 } \\
\hline \multicolumn{4}{|l|}{ Qualité(s) *๑ } \\
\hline \\
\hline \multirow{2}{*}{\multicolumn{4}{|c|}{$\begin{array}{l}\text { D c02 - Analyse de problèmes } \\
\text { c03 - Investigation }\end{array}$}} \\
\hline & & & \\
\hline \multicolumn{4}{|l|}{$\square$ Q04-Conception } \\
\hline \\
\hline \multirow{2}{*}{\multicolumn{4}{|c|}{$\begin{array}{l}\square \text { Q06 - Travail individuel et en équipe } \\
\square \text { Q07 - Communication }\end{array}$}} \\
\hline & & & \\
\hline \multicolumn{4}{|c|}{$\square$ C08 - Professionnalisme } \\
\hline \multirow{2}{*}{\multicolumn{4}{|c|}{$\begin{array}{l}\square 00 \text { - Impact du génie sur la sociáté et renvironnement } \\
\square 010 \text { - Déontologe é égutén }\end{array}$}} \\
\hline & & & \\
\hline \multirow{2}{*}{\multicolumn{4}{|c|}{$\begin{array}{l}\square \text { Q11- Économie et gestion de projets } \\
\square \text { Q12 - Apprentissage continu }\end{array}$}} \\
\hline & & & \\
\hline \multicolumn{4}{|l|}{ Mode d'évaluation *0 } \\
\hline \multicolumn{4}{|l|}{ Individuelle } \\
\hline \multicolumn{4}{|l|}{ Mode de réponse $=\bullet$} \\
\hline \multicolumn{2}{|l|}{ Professeur seulement } & & $\checkmark$ \\
\hline \multicolumn{4}{|l|}{ Système de points * $\bullet$} \\
\hline \multicolumn{2}{|l|}{ Sur 20} & & $\checkmark$ \\
\hline \multicolumn{4}{|l|}{ Diffusion $\ominus$} \\
\hline \multicolumn{2}{|l|}{ Privée } & & $\checkmark$ \\
\hline \multicolumn{4}{|l|}{ Enregistrer } \\
\hline \multicolumn{2}{|c|}{$\begin{array}{l}\text { Programmes | Qualités | Niveaux de développenent } \\
\text { AIDE }\end{array}$} & & $\begin{array}{l}\text { TECHNIQUE }(\odot)= \\
\text { MONTRÉAL }\end{array}$ \\
\hline
\end{tabular}

Une fois les éléments de compétences précisés, il est possible d'ajouter les critères d'évaluation comme montré à la figure 4 . 


\section{Figure 4}

Identification des éléments de compétences et des critères d'évaluation

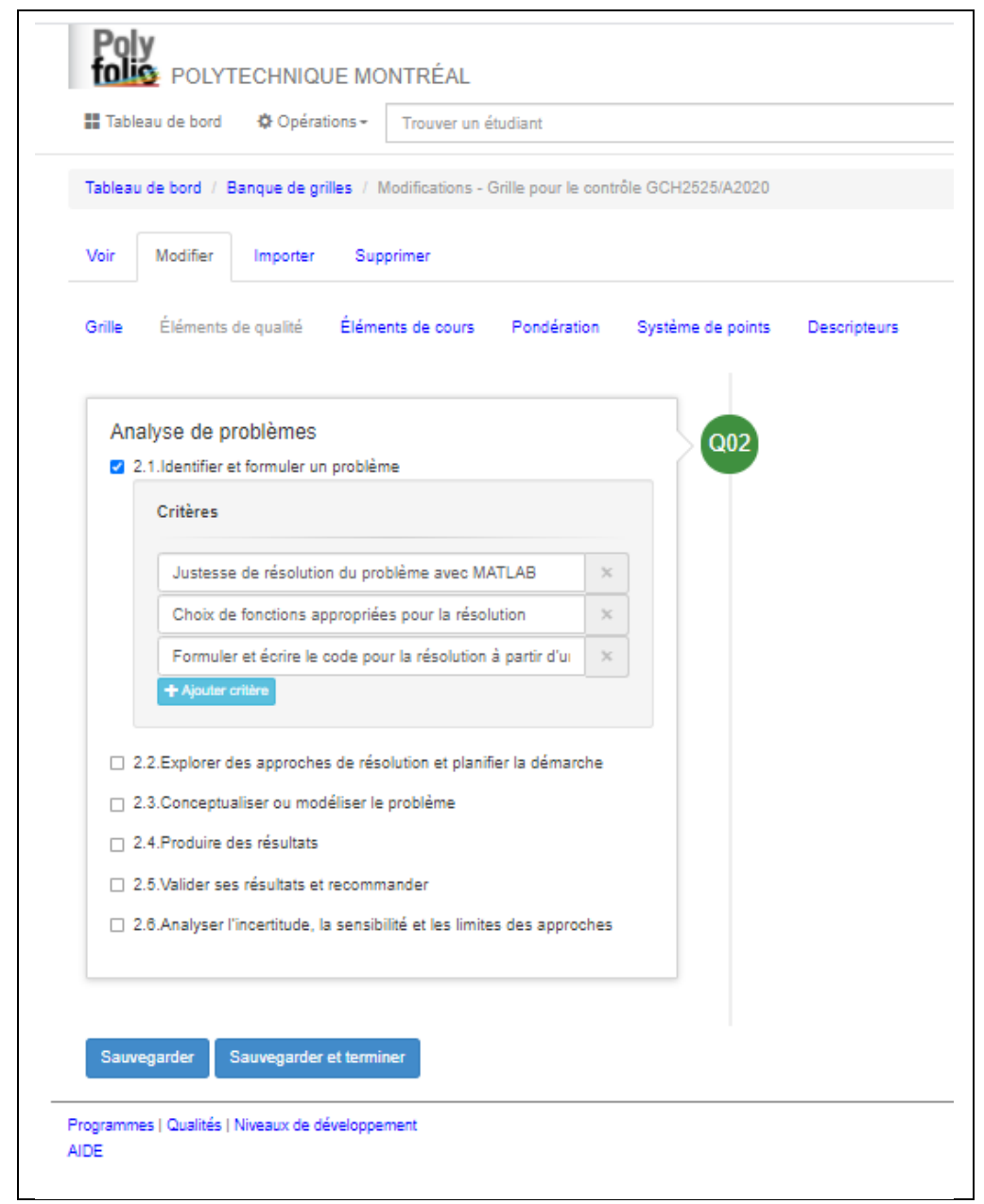

L'enseignant indique également la pondération de chacun des éléments énumérés. Il est important de mentionner que le comité responsable du processus de conception de Polyfolio avait anticipé le risque d'une multiplication des évaluations, causant une surcharge de travail, autant pour les étudiants que pour les enseignants. C'est pourquoi il lui a semblé pertinent d'envisager la possibilité qu'une même activité d'évaluation puisse servir à la fois pour documenter le parcours du développement des compétences du programme (ce sont les informations consignées dans Polyfolio) et pour la note du cours dans lequel se déroule le contrôle des acquis. Cette décision s'est avérée fort appréciée.

Une fois ces opérations réalisées, il reste à préciser les descripteurs pour chacune des cellules de la grille. Polytechnique Montréal a choisi d'utiliser une grille avec une échelle d'acquisition à quatre échelons. Deux de ces niveaux correspondent à la réussite dans la progression du développement de la compétence, soit les niveaux « conforme au niveau attendu » et " supérieur au niveau attendu ». Les deux autres niveaux correspondent à l'échec, soit « sous le niveau attendu » ou encore " clairement sous le niveau attendu ». Un exemple d'une grille d'évaluation critériée complète est présenté à la figure 5. 


\section{Figure 5}

Grille d'évaluation critériée complétée

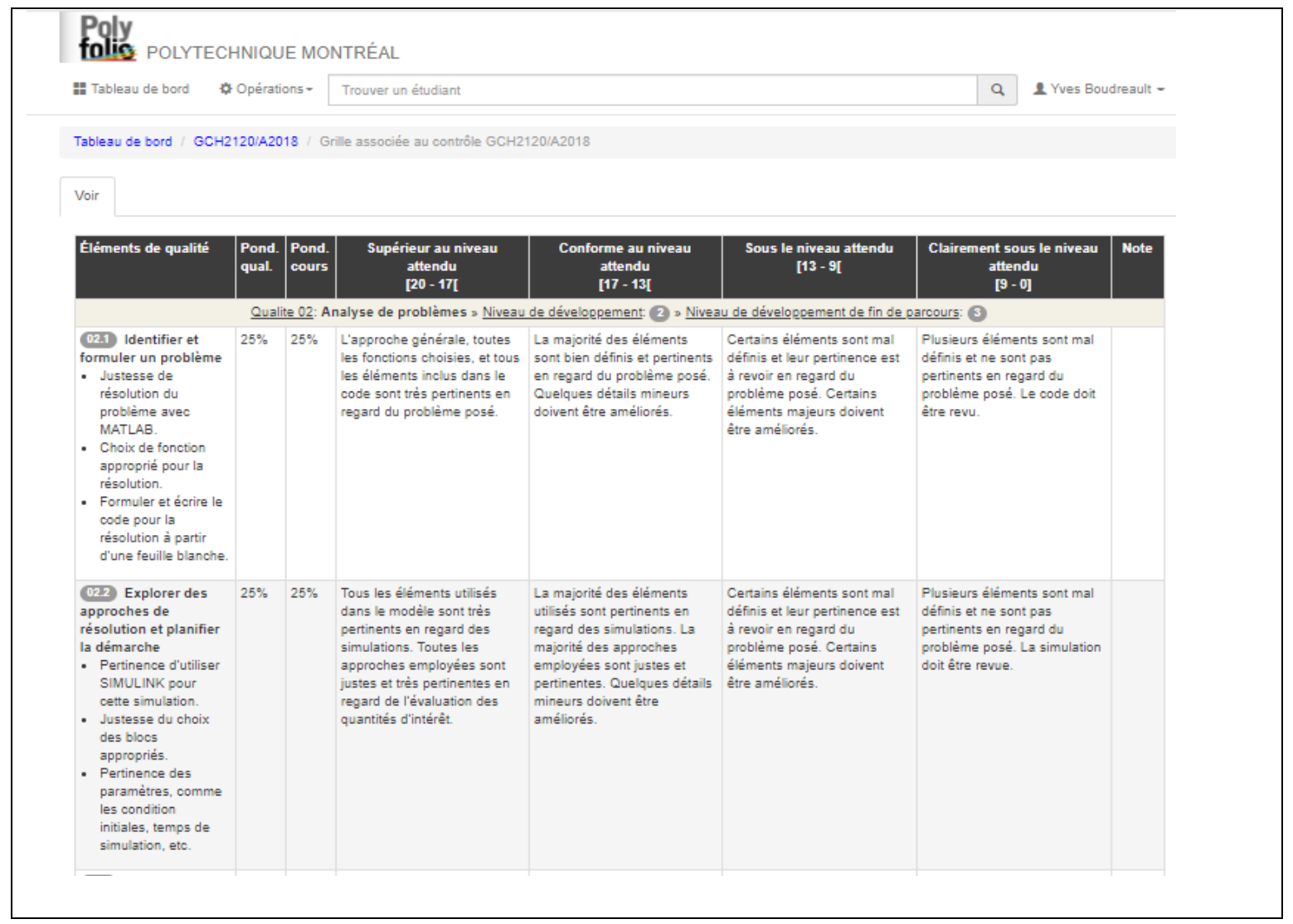

Il faut mentionner, cependant, que l'échec de la compétence ne mène pas nécessairement à un échec au cours, puisque d'autres activités d'évaluation peuvent avoir lieu. De plus, la réussite des compétences n'est pas une condition prescriptive, puisque le BCAPG évalue la qualité des programmes et non les étudiants. Chaque responsable de programme établit donc des cibles de réussite des compétences et prend les mesures nécessaires pour améliorer le programme en fonction de ces cibles. Par exemple, un programme peut s'être donné une cible initiale de réussite de l'ordre de $75 \%$ des étudiants du programme. Une amélioration peut ainsi être observée au prochain cycle d'évaluation pour l'agrément du programme s'il y a atteinte ou augmentation de ce pourcentage. Cette cible constitue l'un des éléments de l'amélioration continue des programmes.

Pour corriger les travaux et attribuer une note aux étudiants réalisant un contrôle des acquis, l'enseignant doit, à partir de son tableau de bord, cliquer sur la section de cours désirée pour que la liste des étudiants apparaisse. La figure 6 montre la liste des étudiants d'un contrôle où un bouton " Actions " présente les opérations possibles pour chaque étudiant. 


\section{Figure 6}

Entrer des résultats par étudiant

\begin{tabular}{|c|c|c|c|c|c|c|c|c|}
\hline$\square$ & Cours & Section d'origine $\wedge$ & Matricule ^ & Prénom / Nom ^ & Programme $\wedge$ & $\begin{array}{l}\text { Nombre de } \\
\text { résultats }\end{array}$ & 0 & \\
\hline$\square$ & $01 \mathrm{C}$ & GCH3100C-01C & 1804509 & & BICHE & $0 / 6$ & Actions - & \\
\hline ○ & $01 \mathrm{C}$ & $\mathrm{GCH} 3100 \mathrm{C}-01 \mathrm{C}$ & 1837642 & & BICHE & $0 / 6$ & \multirow{2}{*}{\multicolumn{2}{|c|}{$\begin{array}{l}\text { Remplir la grille } \\
\text { L Gérer les fichiers bcap }\end{array}$}} \\
\hline$\square$ & $01 \mathrm{C}$ & GCH $3100 \mathrm{C}-01 \mathrm{C}$ & 1837653 & & BICHE & $0 / 6$ & & \\
\hline$\square$ & $01 \mathrm{C}$ & $\mathrm{GCH} 3100 \mathrm{C}-01 \mathrm{C}$ & 1837844 & & BICHE & $0 / 6$ & \multirow{2}{*}{\multicolumn{2}{|c|}{$\begin{array}{l}\text { Consulter les notes } \\
\text { 国 Voir le Tableau de parc } \\
\text { nuturis. }\end{array}$}} \\
\hline$\square$ & $01 \mathrm{C}$ & GCH3100C-01C & 1854055 & & BICHE & $0 / 6$ & & \\
\hline$\square$ & $01 \mathrm{C}$ & GCH3100C-01C & 1856834 & & BICHE & $0 / 6$ & Actions = & \\
\hline$\square$ & $01 \mathrm{C}$ & $\mathrm{GCH} 3100 \mathrm{C}-01 \mathrm{C}$ & 1870795 & & $\mathrm{BICHE}$ & $0 / 6$ & Actions - & \\
\hline$\square$ & $01 \mathrm{C}$ & GCH3100C-01C & 1876948 & & BICHE & $0 / 6$ & Actions - & \\
\hline
\end{tabular}

L'opération « Remplir la grille » affiche la grille destinée à l'étudiant sélectionné et l'enseignant peut alors saisir les résultats directement dans la grille. Une fois les grilles remplies, l'enseignant peut les afficher pour que les étudiants puissent consulter leurs résultats et prendre connaissance de toute rétroaction émise par l'enseignant. Par ailleurs, les enseignants peuvent télécharger les résultats à l'aide d'un fichier Excel, ce qui est fort utile lorsque les notes servent également pour le cours.

\section{Vue étudiante}

Pour l'étudiant, lors de sa première visite dans l'environnement Polyfolio, il est invité à remplir un questionnaire de positionnement personnel (voir figure 7) afin d'y découvrir les 12 compétences, d'évaluer leur importance dans la carrière d'un ingénieur et de s'autoévaluer quant à leur acquisition. Aucune autre fonctionnalité ne lui est accessible tant que ce questionnaire n'est pas rempli. 


\section{Figure 7}

Invitation à remplir le questionnaire de positionnement personnel

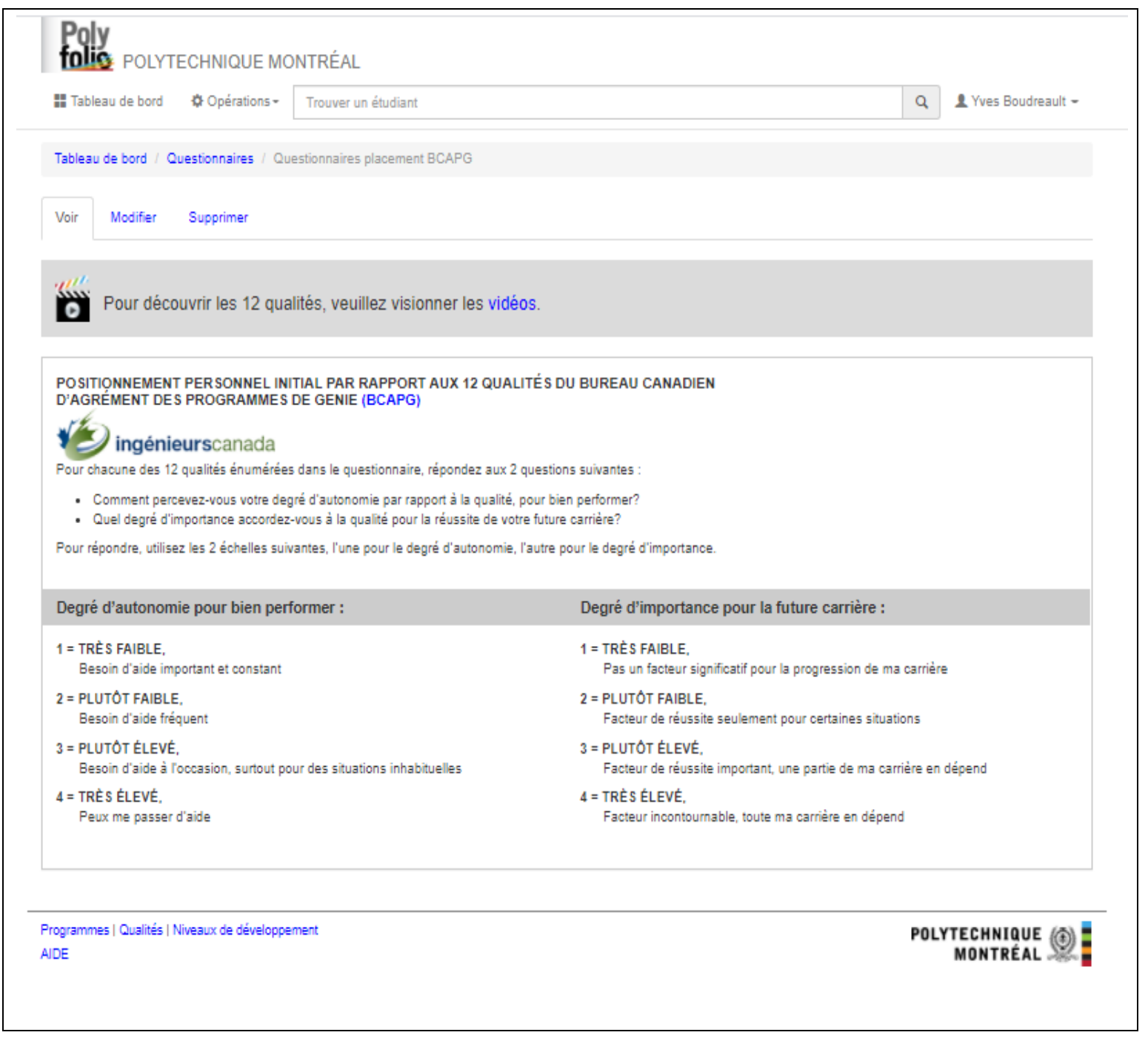

Une fois le questionnaire rempli, l'étudiant peut consulter son « Tableau de parcours » de Polyfolio, dans lequel apparaissent les 12 compétences ainsi que les points de contrôle des acquis de son programme (voir figure 8). 


\section{Figure 8}

Tableau de parcours d'un étudiant

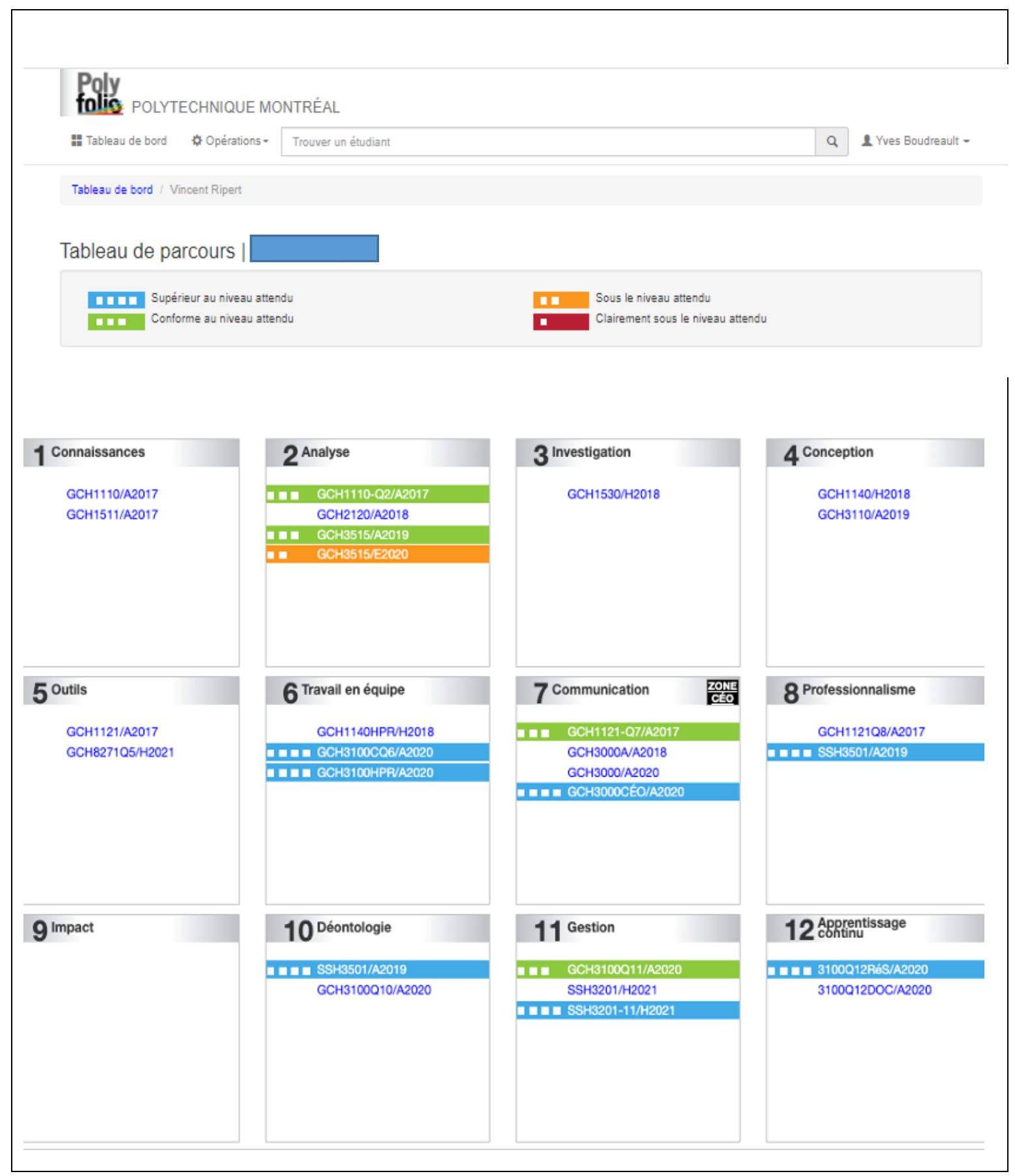

À mesure que les activités sont achevées, un code de couleur (et de petits carrés pour les daltoniens) se juxtapose sur le sigle du cours concerné. Ce code indique la réussite ou l'échec de l'étudiant quant à 
l'atteinte des compétences selon l'échelle d'acquisition présente dans les grilles d'évaluation : bleu pour un résultat supérieur au niveau attendu, vert pour un résultat conforme au niveau attendu, orange pour un résultat sous le niveau attendu et rouge pour un résultat clairement sous le niveau attendu. Le caractère visuel du tableau de parcours offre à l'étudiant une vue d'ensemble instantanée de son parcours, une sorte de rétroaction visuelle globale qui l'encourage à poursuivre lorsqu'il réussit ou à éventuellement corriger le tir pour les compétences non réussies lors des contrôles des acquis. Ce dernier aspect constitue l'élément réflexif du portfolio, où les étudiants qui obtiennent un code rouge ou orange peuvent être invités à produire un texte de réflexion sur les raisons de leur échec et les mesures à entreprendre pour s'améliorer. Toutefois, c'est chaque programme qui décide s'il rend obligatoire cette réflexion.

\section{Résultats et discussion}

Depuis 2017, tous les étudiants utilisent Polyfolio durant leurs études de baccalauréat à Polytechnique. Toutefois, l'appropriation de Polyfolio par les enseignants et les étudiants ne s'est pas réalisée facilement. La résistance à l'utilisation de Polyfolio a été difficile à surmonter, surtout lorsque l'environnement a souffert d'instabilité lors des deux premières années de mise en production. Malgré ces difficultés, son usage fait maintenant partie des pratiques évaluatives courantes, notamment pour le suivi et l'évaluation des compétences. Polyfolio est un outil numérique polyvalent qui contribue à la formation des ingénieurs dans un contexte de programmes fondés sur un référentiel de compétences.

Ce sont en grande majorité les enseignants qui donnent les cours identifiés par le programme servant pour l'évaluation des compétences qui utilisent Polyfolio. Pour les enseignants, l'évaluation des compétences est souvent perçue comme une tâche supplémentaire où ils doivent exploiter de nouveaux mécanismes d'évaluation avec lesquels ils étaient peu familiers. Par exemple, le recours à des évaluations en situation authentique, à des projets de plus ou moins grande envergure ou encore à des grilles critériées devenait pertinent dans le contexte d'évaluations de compétences qui sont définies par le programme de formation (Tardif, 2006). Ces pratiques évaluatives étaient nouvelles pour une majorité de ces enseignants, ces derniers étant plutôt habitués à évaluer des connaissances et des contenus relatifs à leurs cours. Le Bureau d'appui et d'innovation pédagogique (BAIP) de Polytechnique a dû animer plusieurs ateliers portant sur ces thèmes et offrir des accompagnements aux enseignants qui en avaient besoin. Par ailleurs, la rédaction d'un manuel de l'utilisateur pour l'outil Polyfolio ainsi que les séances de formation auprès des enseignants et du personnel des départements a atténué grandement cette résistance en démontrant la simplicité d'utilisation de l'outil.

Pour les étudiants, la priorité est la réussite de leurs cours et l'obtention d'un diplôme en ingénierie, plutôt que l'acquisition des compétences, surtout que celle-ci n'est pas prescriptive. À ce sujet, Taylor et al. (2009) ont montré que le recours au portfolio peut avoir un effet positif sur la motivation et l'engagement des étudiants, mais à la condition que l'évaluation soit sommative. À Polytechnique, ce sont pour l'instant les notes obtenues dans les cours qui conduisent à l'obtention du diplôme. Le lien entre les résultats relatifs aux compétences dans Polyfolio et les notes demeure indirect. Ainsi, les commentaires recueillis auprès des étudiants concernant la facilité d'utilisation et l'utilité du portfolio demeurent mitigés. Comme l'a souligné McMullan (2008), c'est surtout l'aspect réflexif inhérent du portfolio qui reçoit des commentaires défavorables, notamment par les étudiants qui mentionnent avoir un faible intérêt pour la rédaction. À ce propos, les commentaires suivants reflètent l'opinion de plusieurs étudiants : " Je trouve que c'est une perte de temps, en plus je ne suis pas sûr que quelqu'un va lire ma réflexion "; " On nous demande de réfléchir à nos compétences, mais il faut ouvrir chacun des cours dans le Polyfolio pour retrouver nos résultats, mais je ne sais pas trop quoi en dire »; "Les résultats dépendent de mon équipe, ce n'est pas tous les étudiants qui travaillent bien, pourquoi dois-je en payer le prix? ». 
Pour les administrateurs et les responsables de programme, Polyfolio a permis d'accélérer le processus de cueillette d'informations exigées pour la rédaction du rapport d'évaluation à remettre au BCAPG lors des visites d'agrément qui surviennent sur un cycle habituel de six ans. Sa principale force est de rendre facilement accessibles les informations pertinentes à la prise de décision en documentant le parcours de développement des compétences.

\section{Liste de références}

Bélisle, M., Cabana, M., Beaucher, C., Lakhal, S. et Leroux, J. L. (2017). Implantation du portfolio numérique pour soutenir le développement des compétences professionnelles : le cas de quatre programmes de formation à l'enseignement. Dans A. Stockless, I. Lepage et P. Plante (dir.), Actes du colloque CIRTA 2017. Ouvrir les murs de la classe avec le numérique. http://archipel.uqam.ca/11142

Bélair, L. et Van Nieuwenhoven, C. (2010). Le portfolio de consignation ou d'évaluation authentique. Dans L. Paquay, C. Van Nieuwenhoven et P. Wouters, L'évaluation du développement professionnel. De Boeck Supérieur.

Bibeau, R. (2007). À chacun son portfolio numérique. Bulletin collégial des technologies de l'information et des communications, 65. https://bulletinclic.profweb.ca/cgi-bin/aff.pl?page=article\&id=2053

Ingénieurs Canada. (2018). Bureau canadien d'agrément des programmes de génie. Normes et procédures d'agrément 2018. https://engineerscanada.ca/sites/default/files/accreditation/Accreditation-criteria-procedures-2018.pdf

McMullan, M. (2008). Using portfolios for clinical practice learning and assessment: the pre-registration nursing student's perspective. Nurse Education Today, 28(7), 873-879. https://doi.org/10.1016/j.nedt.2007.11.006

Prégent, R., Bernard, H. et Kozanitis, A. (2009). Enseigner à l'université dans une approche-programme. Un défi à relever. Montréal : Presses internationales Polytechnique.

Shaklee, B. D., Barbour, N. E., Ambrose, R., et Hansford, S. J. (1997). Designing and using portfolios. Boston: Allyn and Bacon.

Tardif, J. (2006). L'évaluation des compétences: documenter le parcours de développement. Montréal : Chenelière éducation.

Taylor, C., Stewart, L., et Bidewell, J. (2009). Nursing students' appraisal of their professional portfolios in demonstrating clinical competence. Nurse Educator, 34(5), 217-222. https://doi.org/10.1097/NNE.0b013e3181b2b530 